\title{
三氟甲基酰腙与烯丙基硅或烯丙基嗍试剂的烯丙基化反应研究
}

\author{
虎永琴黄丹凤* 王克虎赵转霞 \\ 赵芳霞徐炜刚胡雨来* \\ (西北师范大学化学化工学院 兰州 730070)
}

\begin{abstract}
摘要 探索了在路易斯酸存在下，三氟甲基酰腙与烯丙基三甲基硅烷或烯丙基硼酸频哪醇酯的烯丙基化反应，高产率 地获得了一系列三氟甲基取代的高烯丙基酰肼类化合物. 研究结果表明，在三氟甲基酰腙的烯丙基化反应中，烯丙基 嗍酸频哪醇酯比烯丙基三甲基硅烷的活性高.
\end{abstract}

关键词 三氟甲基酰腙; 烯丙基三甲基硅烷; 烯丙基硼酸频哪醇酯; 烯丙基化; 三氟甲基高烯丙基酰肼

\section{Study on Allylation Reactions of Trifluoromethylated Acylhydrazones with Allylsilanes or Allylboronates}

\author{
Hu, Yongqin Huang, Danfeng* Wang, Kehu Zhao, Zhuanxia \\ Zhao, Fangxia Xu, Weigang Hu, Yulai* \\ (College of Chemistry and Chemical Engineering, Northwest Normal University, Lanzhou 730070)
}

\begin{abstract}
Allylation reactions of trifluoromethylated acylhydrazones with allyltrimethylsilane or pinacolyl allylboronate were found to proceed smoothly in the presence of Lewis acid to afford a series of trifluoromethylated homoallylic $\mathrm{N}$-acylhydrazines with high yields. The results showed that the activity of pinacolyl allylboronate was higher than that of allyltrimethylsilane in allylation of trifluoromethylated acylhydrazones.

Keywords trifluoromethylated acylhydrazone; allyltrimethylsilane; pinacolyl allylboronate; allylation; trifluoromethylated homoallylic $N$-acylhydrazine
\end{abstract}

作为稳定的亚胺替代物, 酰腙可以和许多亲核试剂 发生反应，生成各种含氮化合物，常见的反应主要有酰 腙的还原反应、Mannich 反应、烯丙基化反应、氭基化 反应和环加成反应以及自由基反应等 ${ }^{[1,2]}$. 其中, 酰腙的 烯丙基化反应是合成高烯丙基酰肼及其衍生物的重要 方法. 近年来, 人们认识到酰肼化合物不仅可以在有机 合成中作为重要的合成砌块 ${ }^{[3]}$, 而且由于这类物质本身 具有很好的生物活性 ${ }^{[4]}$, 它们还可以被用作杀虫剂或抗 菌抗癌药, 甚至是合成一些液晶材料的中间体 ${ }^{[5]}$ 等. 因 此, 探索合成结构多样的酰胇化合物十分必要. 在通过 酰腙的烯丙基化反应合成高烯丙基酰朋类化合物的方 法中, 运用烯丙基有机金属试剂, 如锡 ${ }^{[6]}$ 、铟 ${ }^{[7]}$ 、嶰 ${ }^{[8]}$ 和 硅 ${ }^{[9]}$ 等金属的烯丙基试剂等作为亲核试剂, 是常用的合 成策略.
三氟甲基是一种常见的含氟基团, 将其引入到化合 物分子中能够显著改变化合物的酸碱性、亲脂亲油性以 及代谢稳定性等 ${ }^{[10]}$. 因此，含三氟甲基的化合物被广泛 应用于医药、农药以及材料科学领域中 ${ }^{[11]}$. 在众多的合 成三氟甲基化合物的方法中 ${ }^{[12,13]}$ ，三氟甲基合成砌块法 是一种常用的简便方法 ${ }^{[14]}$. 近年来, 我们课题组发现由 三氟乙醛甲基半缩醛和酰肼生成的三氟甲基酰腙 1 具有 制备简单及性质稳定等特点, 将此酰腙作为三氟甲基合 成砌块, 合成了一系列具有潜在生物活性的三氟甲基含 氮化合物, 取得了较好的反应结果 ${ }^{[15,16]}$. 早在 2009 年, 含有三氟甲基的酰肼化合物被报道具有抗癌作用 ${ }^{[17]}$, 所以探索这类化合物的合成方法具有重要意义. 在本工 作中，我们通过探索三氟甲基酰腙 $\mathbf{1}$ 与烯丙基有机金属 试剂的烯丙基化反应，提供了一种合成含三氟甲基的高

* Corresponding author. E-mail: huyl@nwnu.edu.cn; huangdf@nwnu.edu.cn Received December 4, 2019; revised February 17, 2020; published online February 29, 2020. Project supported by the National Natural Science Foundation of China (No. 21662030). 国家自然科学基金(No. 21662030)资助项目. 
烯丙基酰肼化合物的新方法. 在各种烯丙基有机金属试 剂中, 由于烯丙基硅廉价易得, 烯丙基硼具有官能团兼 容能力, 而且二者都是低毒稳定的试剂 ${ }^{[18,19]}$, 所以我们 选用了烯丙基硅和烯丙基硼作为三氟甲基酰腙 $\mathbf{1}$ 的烯丙 基化试剂.

\section{1 结果与讨论}

\section{1 三氟甲基酰腙与烯丙基三甲基硅烷的烯丙基化反} 应

首先, 探索了在三氟化嗍乙醚(1.0 equiv. $)^{[20]}$ 存在下, 三氟甲基酰腙(1a, 1.0 equiv.) 与烯丙基三甲基硅烷(2a, 1.0 equiv.)的反应. 结果发现, 该反应在 1,2-二氯乙烷中 回流 $18 \mathrm{~h}$ 后, 以 $45 \%$ 的收率得到了目标产物 $3 \mathbf{a}$ (表 1 , Entry 1). 为了进一步提高 $\mathbf{3 a}$ 的产率, 对该反应的物料 比进行了优化(表 1, Entries 2６). 研究表明, 三氟甲基 酰腙(1a)、烯丙基三甲基硅烷(2a)和三氟化硼乙醚的物 质的量比为 $1: 3: 1.5$ 时, $3 \mathbf{a}$ 的产率最高, 可达 $85 \%$ (表 1, Entry 6). 然后, 在此条件下, 对该反应的溶剂进行了 考察(表 1 , Entries 7〜12). 从表 1 可知, 当用二氯甲烷、

表 1 合成化合物 3a 的反应条件优化 ${ }^{a}$

Table 1 Optimization of the reaction conditions for the synthesis of compound 3a

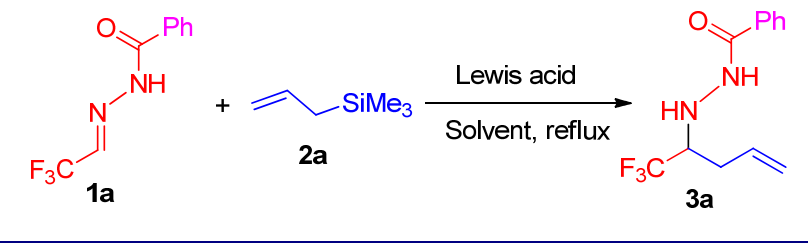

\begin{tabular}{|c|c|c|c|c|}
\hline Entry & $\begin{array}{c}\text { Molar ratio of } \\
\mathbf{1 a} / \mathbf{2 a} / \text { Lewis } \\
\text { acid }\end{array}$ & Lewis acid & Solvent & $\begin{array}{l}\text { Isolated } \\
\text { yield/\% }\end{array}$ \\
\hline 1 & $1 / 1 / 1$ & $\mathrm{BF}_{3} \cdot \mathrm{OEt}_{2}$ & DCE & 45 \\
\hline 2 & $1 / 1 / 1.5$ & $\mathrm{BF}_{3} \bullet \mathrm{OEt}_{2}$ & DCE & 53 \\
\hline 3 & $1 / 1.5 / 1.5$ & $\mathrm{BF}_{3} \cdot \mathrm{OEt}_{2}$ & DCE & 62 \\
\hline 4 & $1 / 2 / 1.5$ & $\mathrm{BF}_{3} \bullet \mathrm{OEt}_{2}$ & DCE & 75 \\
\hline 5 & $1 / 2.5 / 1.5$ & $\mathrm{BF}_{3} \cdot \mathrm{OEt}_{2}$ & DCE & 79 \\
\hline 6 & $1 / 3 / 1.5$ & $\mathrm{BF}_{3} \bullet \mathrm{OEt}_{2}$ & DCE & 85 \\
\hline 7 & $1 / 3 / 1.5$ & $\mathrm{BF}_{3} \bullet \mathrm{OEt}_{2}$ & $\mathrm{CH}_{2} \mathrm{Cl}_{2}$ & 65 \\
\hline 8 & $1 / 3 / 1.5$ & $\mathrm{BF}_{3} \cdot \mathrm{OEt}_{2}$ & $\mathrm{CHCl}_{3}$ & 68 \\
\hline 9 & $1 / 3 / 1.5$ & $\mathrm{BF}_{3} \bullet \mathrm{OEt}_{2}$ & $\mathrm{CH}_{3} \mathrm{CN}$ & 0 \\
\hline 10 & $1 / 3 / 1.5$ & $\mathrm{BF}_{3} \cdot \mathrm{OEt}_{2}$ & $\mathrm{EtOH}$ & 0 \\
\hline 11 & $1 / 3 / 1.5$ & $\mathrm{BF}_{3} \cdot \mathrm{OEt}_{2}$ & 1,4-Dioxane & 0 \\
\hline 12 & $1 / 3 / 1.5$ & $\mathrm{BF}_{3} \cdot \mathrm{OEt}_{2}$ & THF & 0 \\
\hline 13 & $1 / 3 / 1.5$ & $\mathrm{Ni}(\mathrm{ClO})_{4} \cdot 6 \mathrm{H}_{2} \mathrm{O}$ & DCE & 0 \\
\hline 14 & $1 / 3 / 1.5$ & $\mathrm{Zn}(\mathrm{OTf})_{2}$ & DCE & 0 \\
\hline 15 & $1 / 3 / 1.5$ & $\mathrm{Cu}(\mathrm{OTf})_{2}$ & DCE & 0 \\
\hline 16 & $1 / 3 / 1.5$ & $\operatorname{In}(\mathrm{OTf})_{3}$ & DCE & 0 \\
\hline 17 & $1 / 3 / 1.5$ & $\operatorname{In}(\mathrm{OTf})_{3}$ & $\mathrm{CH}_{3} \mathrm{CN}$ & 80 \\
\hline 18 & $1 / 3 / 0$ & - & DCE & 0 \\
\hline
\end{tabular}

${ }^{a}$ All reactions were carried out by using $0.3 \mathrm{mmol}$ of $1 \mathrm{a}, 0.9 \mathrm{mmol}$ of $2,0.45$ mmol of Lewis acid, $5 \mathrm{~mL}$ of solvent, under reflux for $18 \mathrm{~h}$.
氯仿为溶剂时, $\mathbf{3 a}$ 的产率有所降低; 当用乙腈、乙醇、 1,4-二氧六环等为溶剂时，反应不能发生。最后，考察 了其他路易斯酸对反应的影响. 结果发现, 在 $\mathrm{Ni}(\mathrm{ClO})_{4} \bullet$ $6 \mathrm{H}_{2} \mathrm{O} 、 \mathrm{Cu}(\mathrm{OTf})_{2} 、 \mathrm{Zn}(\mathrm{OTf})_{2}$ 或 $\mathrm{In}(\mathrm{OTf})_{3}$ 等金属路易斯酸 存在下, 该反应在 $1,2-$ 二氯乙烷溶剂中都不能发生 $($ 表 1 , Entries $13 \sim 16)$. 但是, 当用 $\operatorname{In}(\mathrm{OTf})_{3}$ 作为促进剂时, 在 乙腈溶剂中, 反应能够发生, 3a 的产率可达 $80 \%$ (表 1, Entry 17). 需要说明的是, 在不添加路易斯酸的情况下, 该反应不能发生(表 1, Entry 18). 因此，路易斯酸在反应 中是必需的. 综上所述, 合成化合物 $\mathbf{3 a}$ 的最佳反应条件 是: 三氟甲基酰腙(1a)、烯丙基三甲基硅烷(2a)和三氟化 硼乙醚的物质的量的比为 $1: 3: 1.5$, 溶剂为 $1,2-$ 二氯 乙烷，反应温度为回流.

为了探索该反应对底物的适应性, 在上述最佳反应 条件下，对各种不同官能团取代的三氟甲基酰腙进行了 考察, 结果列于表 2 . 由表 2 可知, 对于芳香酰肼形成的 三氟甲基酰腙来说，取代基在苯环上的位置对反应有较 大影响. 当取代基在苯环的对位和间位时, 产物的产率 较高; 而取代基在苯环的邻位时, 产物的产率较低 $(3 \mathbf{b} \sim 3 \mathbf{d}, 3 \mathbf{3} \sim 3 \mathbf{h})$. 例如，3-甲基苯甲酰肼和 4-甲基苯甲 酰肼形成的三氟甲基酰腙，反应后所得高烯丙基酰肼 3c 和 3d 的产率分别为 75\% 和 85\%; 而 2-甲基苯甲酰肼 形成的三氟甲基酰腙，反应后所得产物 $3 \mathrm{~b}$ 的产率只有 $42 \%$. 研究结果表明，三氟甲基酰腙的芳环上取代基的 电子性质对该反应也有一定的影响. 例如, 对于取代基 在芳环对位上的三氟甲基酰腙来说，不管取代基是供电 子的甲基或甲氧基，还是卤素(氟，氯，溴), 所得产物的 产率都能达到 $75 \%$ 以上 $(\mathbf{3 d} \sim \mathbf{3 e}, \mathbf{3 h} \sim \mathbf{3 i}, \mathbf{3 k})$; 但是当取 代基为强吸电子的三氟甲基时，所得产物 31 的产率只有 $63 \%$. 当用菜酰肼和杂芳酰肼形成的三氟甲基酰腙作为 底物时, 反应也能顺利进行，产率最高可达 $86 \%(3 \mathrm{~m} \sim$ 3o). 但是, 当用噻吩并 $[3,2-b]$ 噻吩-2-甲酰肼衍生的三氟 甲基酰腙作为底物时，反应产率有所降低，3p 的产率只 有 57\%. 最后，考察了脂肪酰肼形成的三氟甲基酰腙对 反应的影响. 研究表明, 当三氟甲基酰腙中的烃基 $\mathrm{R}$ 为 环己基和茮基时，反应都能顺利进行，但是当 $\mathrm{R}$ 为异丙 基时, 产率降低 $(3 \mathbf{q} \sim 3 \mathbf{s})$.

为了进一步探索该方法的普适性，又探索了其他烯 丙基硅试剂, 如烯丙基三氯硅烷、烯丙基二甲基氯硅烷 以及烯丙基三甲氧基硅烷与三氟甲基酰腙 $1 \mathrm{a}$ 的反应. 结果发现，烯丙基二甲基氯硅烷也适合该反应，但是产 物 3a 的产率降低，只有 $65 \%$; 而其他两种烯丙基硅试剂 都不能发生反应. 该研究结果表明, 烯丙基三甲基硅烷 比上述三种烯丙基硅试剂活性高，这与文献所述一致 $\left(\right.$ Scheme 1) ${ }^{[19]}$. 
表 2 三氟甲基酰腙与烯丙基三甲基硅烷的烯丙基化反应 ${ }^{a, b}$

Table 2 Allylation reactions of trifluoromethylated acylhydrazones with allyltrimethylsilane

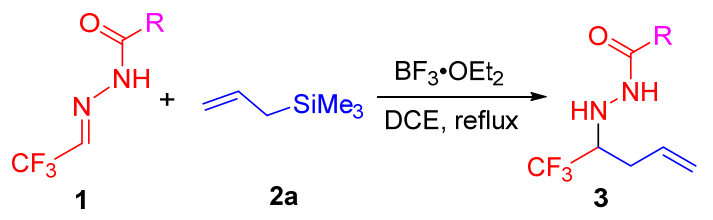

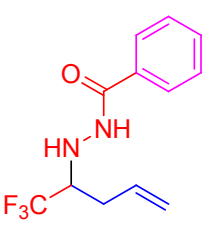

3a: $85 \%$

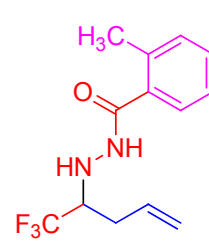

3b: $42 \%$

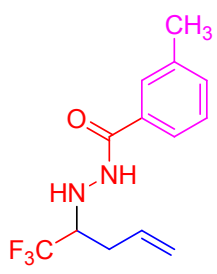

3c: $75 \%$<smiles>C=CCC(NNC(=O)c1ccc(C)cc1)C(F)(F)F</smiles>

3d: $85 \%$<smiles>C=CCC(NC(=O)c1ccc(OC)cc1)C(F)(F)F</smiles>

3e: $75 \%$

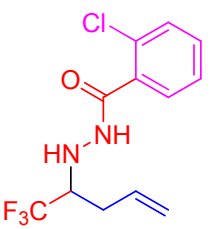

3f: $32 \%$<smiles>C=CCC(NC(=O)c1cccc(Cl)c1)C(F)(F)F</smiles>

3 g: $82 \%$

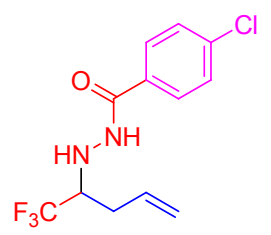

3h: $86 \%$

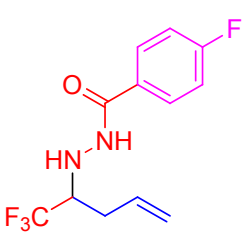

3i: $81 \%$<smiles>C=CCC(NC(=O)c1cccc(Br)c1)C(F)(F)F</smiles>

3j: $72 \%$<smiles>C=CCC(NNC(=O)c1ccc(Br)cc1)C(F)(F)F</smiles>

3k: $80 \%$

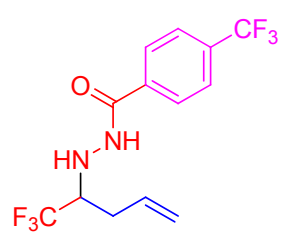

3I: $63 \%$<smiles>C=CCC(NNC(=O)c1ccc2ccccc2c1)C(F)(F)F</smiles>
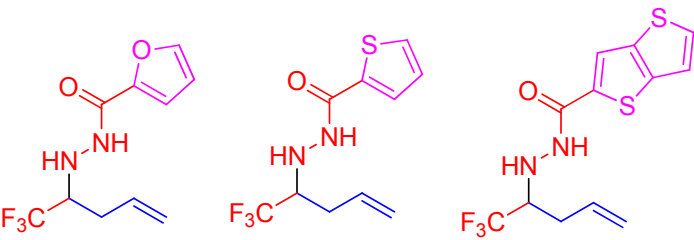

3m: $84 \%$

3n: $86 \%$

3o: $84 \%$

3p: $57 \%$
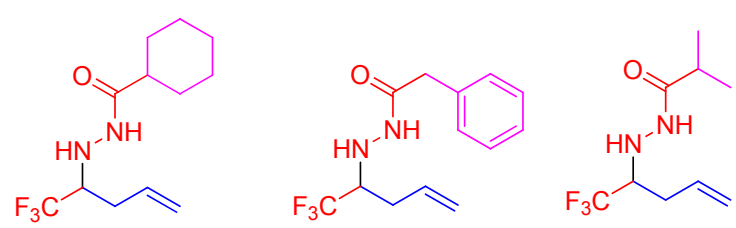

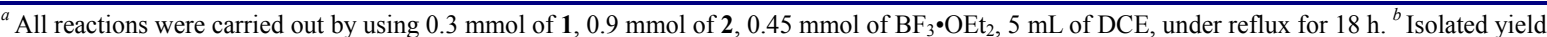

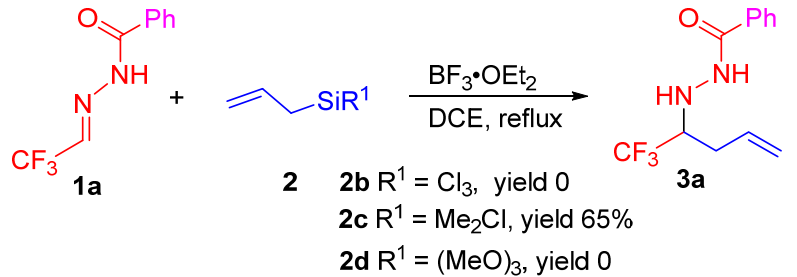

图式 1 烯丙基硅试剂的适用范围

Scheme 1 Substrate scope of allylsilanes

在文献报道 ${ }^{[20]}$ 和实验结果基础上, 我们认为在三 氟化嗍乙醚存在下，三氟甲基酰腙与烯丙基三甲基硅烷 的反应机理可能如 Scheme 2 所示: 首先, 在三氟化嗍乙 醚作用下, 三氟甲基酰腙 $\mathbf{1}$ 与烯丙基三甲基硅烷 $\mathbf{2}$ 分别 形成了二氟嗍一酰腙络合物 5 和有机硅化合物 $\mathbf{6}$; 然后, 6 与二氟硽-酰腙络合物 5 发生烯丙基化反应产生 7; 最 后, 7 经水解产生目标产物 $\mathbf{3}$, 并伴随有三甲基硅醇 $\left(\mathrm{Me}_{3} \mathrm{SiOH}\right)$ 生成.

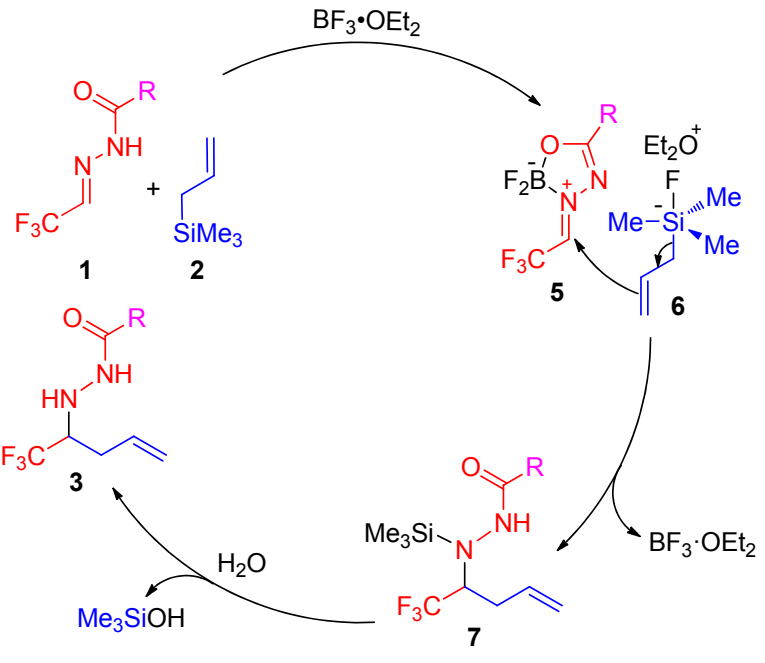

图式 2 可能的反应机理

Scheme 2 Proposed mechanism 


\section{2 三氟甲基酰腙与烯丙基硼酸频哪醇酯的烯丙基化 反应}

虽然在三氟甲基酰腙与烯丙基三甲基硅烷的烯丙 基化反应中取得了较好结果, 但是上述反应也存在如下 缺陷: 反应需要使用过量的烯丙基三甲基硅烷(3 equiv.) 和化学计量的路易斯酸(1.5 equiv.), 反应时间较长等, 这说明烯丙基硅试剂的反应活性不是很高 ${ }^{[21]}$. 为了更 好地探索该反应，我们用烯丙基硼试剂(4)代替烯丙基 三甲基硅烷进行三氟甲基酰腙 $\mathbf{1}$ 的烯丙基化反应研究, 反应结果见表 3. 由表 3 可知, 在三氟化嗍乙醚存在下, 三氟甲基酰腙(1a)与烯丙基硼酸频哪醇酯在上述反应条 件下, 回流反应 $18 \mathrm{~h}$ 后, 仅有 $5 \%$ 的 3a 生成; 经过简单 的反应条件优化 (如反应物的物质的量的比和溶剂等) 后, 3a 的产率被提高到 56\%(表 3, Entries 1,2). 接着, 又 探索了其他路易斯酸如 $\mathrm{Zn}(\mathrm{OTf})_{2} 、 \mathrm{Cu}(\mathrm{OTf})_{2}$ 和 $\mathrm{In}(\mathrm{OTf})_{3}$ 等对该反应的影响(表 3, Entries 3 5). 结果发现, $\mathrm{In}(\mathrm{OTf})_{3}$ 的效果最好, 在 $20 \mathrm{~mol} \% \mathrm{In}(\mathrm{OTf})_{3}$ 存在下, 将三 氟甲基酰腙(1a)与烯丙基硼酸频哪醇酯在乙腈中回流 3 $\mathrm{h}$ 后, 就能以 $90 \%$ 的产率得到 3a. 于是在此条件下, 探

表 3 三氟甲基酰腙与烯丙基硼试剂的烯丙基化反应 ${ }^{a, b}$

Table 3 Allylation of trifluoromethylated acylhydrazones with allylboronates ${ }^{a, b}$

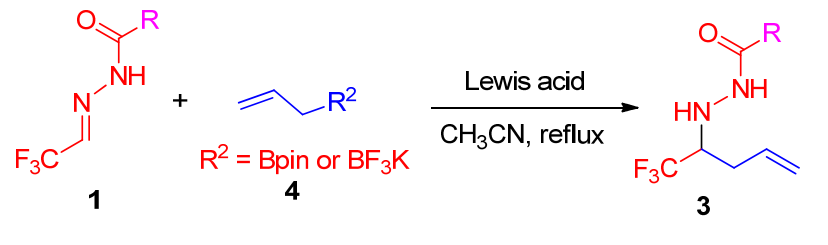

\begin{tabular}{|c|c|c|c|c|c|c|c|}
\hline Entry & $\mathrm{R}$ & $\mathrm{R}^{2}$ & $\begin{array}{c}\text { Molar ratio of } \\
\text { 1/4/Lewis } \\
\text { acid }\end{array}$ & $\begin{array}{c}\text { Lewis } \\
\text { acid }\end{array}$ & Time/h & 3 & $\begin{array}{l}\text { solated } \\
\text { yield } \\
1 \% \\
\end{array}$ \\
\hline $1^{b}$ & $\mathrm{C}_{6} \mathrm{H}_{5}$ & Bpin & $1 / 3.0 / 1.5$ & $\mathrm{BF}_{3} \cdot \mathrm{OEt}_{2}$ & 18 & $\mathbf{3 a}$ & 5 \\
\hline 2 & $\mathrm{C}_{6} \mathrm{H}_{5}$ & Bpin & $1 / 1.5 / 0.2$ & $\mathrm{BF}_{3} \cdot \mathrm{OEt}_{2}$ & 22 & $\mathbf{3 a}$ & 56 \\
\hline 3 & $\mathrm{C}_{6} \mathrm{H}_{5}$ & Bpin & $1 / 1.5 / 0.2$ & $\mathrm{Zn}(\mathrm{OTf})_{2}$ & 22 & $\mathbf{3 a}$ & 26 \\
\hline 4 & $\mathrm{C}_{6} \mathrm{H}_{5}$ & Bpin & $1 / 1.5 / 0.2$ & $\mathrm{Cu}(\mathrm{OTf})_{2}$ & 22 & $\mathbf{3 a}$ & 0 \\
\hline 5 & $\mathrm{C}_{6} \mathrm{H}_{5}$ & Bpin & $1 / 1.5 / 0.2$ & $\operatorname{In}(\mathrm{OTf})_{3}$ & 3 & $\mathbf{3 a}$ & 90 \\
\hline 6 & $p-\mathrm{MeC}_{6} \mathrm{H}_{4}$ & Bpin & $1 / 1.5 / 0.2$ & $\operatorname{In}(\mathrm{OTf})_{3}$ & 3 & $3 d$ & 82 \\
\hline 7 & $p-\mathrm{MeOC}_{6} \mathrm{H}_{4}$ & ${ }_{4}$ Bpin & $1 / 1.5 / 0.2$ & $\operatorname{In}(\mathrm{OTf})_{3}$ & 3 & $3 e$ & 88 \\
\hline 8 & $p-\mathrm{ClC}_{6} \mathrm{H}_{4}$ & Bpin & $1 / 1.5 / 0.2$ & $\operatorname{In}(\mathrm{OTf})_{3}$ & 3 & $3 \mathbf{h}$ & 83 \\
\hline 9 & $p-\mathrm{FC}_{6} \mathrm{H}_{4}$ & Bpin & $1 / 1.5 / 0.2$ & $\operatorname{In}(\mathrm{OTf})_{3}$ & 3 & $3 \mathbf{i}$ & 59 \\
\hline 10 & $m-\mathrm{BrC}_{6} \mathrm{H}_{4}$ & Bpin & $1 / 1.5 / 0.2$ & $\operatorname{In}(\mathrm{OTf})_{3}$ & 3 & $\mathbf{3 j}$ & 67 \\
\hline 11 & $p-\mathrm{CF}_{3} \mathrm{C}_{6} \mathrm{H}_{4}$ & Bpin & $1 / 1.5 / 0.2$ & $\operatorname{In}(\mathrm{OTf})_{3}$ & 3 & 31 & 96 \\
\hline 12 & 2-Naphthyl & Bpin & $1 / 1.5 / 0.2$ & $\operatorname{In}(\mathrm{OTf})_{3}$ & 3 & $3 m$ & 75 \\
\hline 13 & 2-Furyl & Bpin & $1 / 1.5 / 0.2$ & $\operatorname{In}(\mathrm{OTf})_{3}$ & 3 & $3 n$ & 81 \\
\hline 14 & Bithienyl & Bpin & $1 / 1.5 / 0.2$ & $\operatorname{In}(\mathrm{OTf})_{3}$ & 3 & $3 p$ & 63 \\
\hline 15 & $\mathrm{Bn}$ & Bpin & $1 / 1.5 / 0.2$ & $\operatorname{In}(\mathrm{OTf})_{3}$ & 3 & $3 \mathbf{r}$ & 86 \\
\hline 16 & Isobutyl & Bpin & $1 / 1.5 / 0.2$ & $\operatorname{In}(\mathrm{OTf})_{3}$ & 3 & $3 s$ & 64 \\
\hline 17 & $\mathrm{C}_{6} \mathrm{H}_{5}$ & $\mathrm{BF}_{3} \mathrm{~K}$ & $1 / 1.5 / 0.2$ & $\operatorname{In}(\mathrm{OTf})_{3}$ & 3 & $\mathbf{3 a}$ & 84 \\
\hline
\end{tabular}

${ }^{a}$ Reactions were carried out by using $0.3 \mathrm{mmol}$ of $1,0.45 \mathrm{mmol}$ of $4,0.06$ mmol of Lewis acid, $5 \mathrm{~mL}$ of $\mathrm{CH}_{3} \mathrm{CN}$, under reflux. ${ }^{b} 1,2$-dichloroethane was used as solvent.
索了其它酰腙与烯丙基硼酸频哪醇酯的烯丙基化反应 (表 3, Entries 6 16). 结果表明, 各种三氟甲基酰腙与 烯丙基硼酸频哪醇酯的烯丙基化反应都能顺利进行，并 且都以较高产率得到了目标产物. 令人高兴的是，和三 氟甲基酰腙与烯丙基三甲基硅烷的反应相比，烯丙基硼 酸频哪醇酯作为烯丙基化试剂时，大大缩短了反应时 间; 路易斯酸的用量大大减少，由原来的化学计量变为 催化量(20 mol\%); 而且烯丙基硼酸频哪醇酯仅需稍微 过量(1.5 equiv.).一些产物的产率明显提高, 其中, 最 突出的就是产物 31 和 $3 \mathrm{~s}$, 产率分别由原来的 $63 \%$ 和 $32 \%$ 提高到 96\%和 64\%. 这些反应结果表明，在三氟甲基酰 腙的烯丙基化反应中, 烯丙基硼酸频哪醇酯比烯丙基三 甲基硅烷的活性要高. 由表 3 还可知, 当用烯丙基三氟 嗍酸钾作为烯丙基化试剂时, 反应也能顺利进行, 并以 84\%的收率得到了目标产物 3a(表 3, Entry 17).

\section{2 结论}

综上所述，探索了在路易斯酸作用下，三氟甲基酰 腙与烯丙基三甲基硅烷或烯丙基硼酸频哪醇酯的烯丙 基化反应，获得了一系列的三氟甲基取代的高烯丙基酰 肼类化合物. 该反应具有反应条件温和、操作简单和产 率高等特点. 研究结果表明, 在该反应中, 烯丙基硼酸 频哪醇酯比烯丙基三甲基硅烷的活性高. 通过使用制备 简单、性质稳定的三氟甲基酰腙作为三氟甲基合成砌块, 提供了一种合成含有三氟甲基的高烯丙基酰肼类化合 物的新方法, 进一步拓展了以三氟甲基酰腙作为三氟甲 基合成砌块在三氟甲基化反应中的应用范围.

\section{3 实验部分}

\section{1 仪器与试剂}

核磁共振谱用 BRUKER PT jxf790425AM 400 $\mathrm{MHz} / 600 \mathrm{MHz}$ 型核磁共振仪测定, 以気代氯仿作为溶 剂, TMS 为内标; 高分辨质谱用 Bruker APEX II 傅里叶 变换离子回旋共振质谱仪测定, ESI 源; 熔点测定用显 微熔点测定仪测定, 温度未校正. 实验中所用试剂均为 国产分析纯级. 所用溶剂均通过标准方法蒸馏干燥.

\section{2 实验方法}

3.2.1 三氟甲基酰腙与烯丙基三甲基硅烷的烯丙基 化反应

在 $50 \mathrm{~mL}$ 的圆底烧瓶中, 依次加入三氟甲基酰腙 (1a) $(0.065 \mathrm{~g}, 0.3 \mathrm{mmol}) 、 1,2$-二氯乙烷 $(3 \mathrm{~mL}) 、 三$ 氟化硓 乙醚 $(0.064 \mathrm{~g}, 0.45 \mathrm{mmol})$ 和烯丙基三甲基硅烷(2) (103 g, $0.9 \mathrm{mmol}$ ), 将此混合物回流 $18 \mathrm{~h}$, 薄层色谱 (TLC)检测 反应完全. 然后向反应体系中加入饱和氯化钠水溶液 (5 $\mathrm{mL})$ 淬灭反应，反应混合液用乙酸乙酯萃取 $(10 \mathrm{~mL} \times 3)$, 
分出有机相, 经无水硫酸镁干燥, 除去溶剂后进行柱层 析 $[V$ (石油醚 $): V($ 乙酸乙酯 $)=5: 1]$ 分离得到化合物 $\mathbf{3 a}$. 同样方法合成化合物 $\mathbf{3 b} \sim \mathbf{3 s}$.

$N^{\prime}$-(1-三氟甲基丁-3-烯-1-基)苯甲酰肼(3a): $65.9 \mathrm{mg}$, 白色固体, 产率 85\%. m.p. $96 \sim 97{ }^{\circ} \mathrm{C}$ (文献值 ${ }^{[15 \mathrm{a}]}$ : $96 \sim$ $\left.97{ }^{\circ} \mathrm{C}\right) ;{ }^{1} \mathrm{H}$ NMR $\left(400 \mathrm{MHz}, \mathrm{CDCl}_{3}\right) \delta: 7.80(\mathrm{~s}, 1 \mathrm{H}), 7.73$ (d, $J=6.8 \mathrm{~Hz}, 2 \mathrm{H}), 7.54$ (t, $J=7.2 \mathrm{~Hz}, 1 \mathrm{H}), 7.45$ (t, $J=8.0$ $\mathrm{Hz}, 2 \mathrm{H}), 6.06 \sim 5.95(\mathrm{~m}, 1 \mathrm{H}), 5.34(\mathrm{~d}, J=6.0 \mathrm{~Hz}, 1 \mathrm{H})$, $5.30(\mathrm{~s}, 1 \mathrm{H}), 4.94(\mathrm{~d}, J=6.0 \mathrm{~Hz}, 1 \mathrm{H}), 3.54 \sim 3.46(\mathrm{~m}, 1 \mathrm{H})$, $2.62(\mathrm{~d}, J=14.8 \mathrm{~Hz}, 1 \mathrm{H}), 2.37 \sim 2.30(\mathrm{~m}, 1 \mathrm{H}) ;{ }^{13} \mathrm{C} \mathrm{NMR}$ $\left(100 \mathrm{MHz}, \mathrm{CDCl}_{3}\right) \delta: 167.5,132.4,132.2,128.9,127.1$, $126.2\left(\mathrm{q}, \quad J_{\mathrm{C}-\mathrm{F}}=279.0 \mathrm{~Hz}\right), 120.2,60.85\left(\mathrm{q}, J_{\mathrm{C}-\mathrm{F}}=26.0\right.$ $\mathrm{Hz}), 31.7\left(\mathrm{q}, J_{\mathrm{C}-\mathrm{F}}=2.0 \mathrm{~Hz}\right) ;{ }^{19} \mathrm{~F}$ NMR $\left(376 \mathrm{MHz}, \mathrm{CDCl}_{3}\right)$ $\delta$ : $-75.48(\mathrm{~d}, J=3.76 \mathrm{~Hz})$.

$N^{\prime}$-(1-三氟甲基丁-3-烯-1-基)-2-甲基苯甲酰肼(3b): $67.0 \mathrm{mg}$, 白色固体, 产率 $82 \%$. m.p. $79 \sim 80{ }^{\circ} \mathrm{C}$ (文献 值 ${ }^{[15 \mathrm{a}]}$ : $\left.79 \sim 80{ }^{\circ} \mathrm{C}\right) ;{ }^{1} \mathrm{H}$ NMR $\left(400 \mathrm{MHz}, \mathrm{CDCl}_{3}\right) \delta: 7.42$ (s, 1H), $7.36 \sim 7.31(\mathrm{~m}, 2 \mathrm{H}), 7.26 \sim 7.18(\mathrm{~m}, 2 \mathrm{H}), 6.05 \sim$ $5.95(\mathrm{~m}, 1 \mathrm{H}), 5.33$ (d, $J=7.2 \mathrm{~Hz}, 1 \mathrm{H}), 5.30(\mathrm{~s}, 1 \mathrm{H}), 4.91$ (d, $J=6.4 \mathrm{~Hz}, 1 \mathrm{H}), 3.56 \sim 3.48(\mathrm{~m}, 1 \mathrm{H}), 2.62(\mathrm{~d}, J=14.8$ $\mathrm{Hz}, 1 \mathrm{H}), 2.43$ (s, 3H), 2.39 2.31 (m, 1H); ${ }^{13} \mathrm{C}$ NMR $(150$ $\left.\mathrm{MHz}, \mathrm{CDCl}_{3}\right) \delta: 169.6,137.0,133.4,132.1,131.3,130.8$, $127.2,126.2\left(\mathrm{q}, J_{\mathrm{C}-\mathrm{F}}=280.5 \mathrm{~Hz}\right), 126.0,120.3,60.8(\mathrm{q}$, $\left.J_{\mathrm{C}-\mathrm{F}}=27.0 \mathrm{~Hz}\right), 31.7\left(\mathrm{q}, J_{\mathrm{C}-\mathrm{F}}=2.1 \mathrm{~Hz}\right), 19.8 ;{ }^{19} \mathrm{~F}$ NMR $\left(376 \mathrm{MHz}, \mathrm{CDCl}_{3}\right) \delta:-75.30(\mathrm{~d}, J=7.52 \mathrm{~Hz})$.

$N^{\prime}$-(1-三氟甲基丁-3-烯-1-基)-3-甲基苯甲酰肼(3c): $61.3 \mathrm{mg}$, 白色固体, 产率 75\%. m.p. $75 \sim 77{ }^{\circ} \mathrm{C}$ (文献 值 ${ }^{[15 \mathrm{a}]}$ : $\left.75 \sim 77{ }^{\circ} \mathrm{C}\right) ;{ }^{1} \mathrm{H}$ NMR $\left(600 \mathrm{MHz}, \mathrm{CDCl}_{3}\right) \delta: 7.83$ (d, $J=6.0 \mathrm{~Hz}, 1 \mathrm{H}), 7.55(\mathrm{~s}, 1 \mathrm{H}), 7.50(\mathrm{~d}, J=7.2 \mathrm{~Hz}, 1 \mathrm{H})$, $7.34 \sim 7.30(\mathrm{~m}, 2 \mathrm{H}), 6.03 \sim 5.96(\mathrm{~m}, 1 \mathrm{H}), 5.31(\mathrm{~d}, J=10.2$ $\mathrm{Hz}, 1 \mathrm{H}), 5.29$ (s, 1H), 4.93 (d, $J=6.0 \mathrm{~Hz}, 1 \mathrm{H}), 3.51 \sim 3.46$ (m, 1H), 2.61 (d, $J=14.4 \mathrm{~Hz}, 1 \mathrm{H}), 2.38(\mathrm{~s}, 3 \mathrm{H}), 2.37 \sim$ $2.31(\mathrm{~m}, 1 \mathrm{H}) ;{ }^{13} \mathrm{C}$ NMR $\left(150 \mathrm{MHz}, \mathrm{CDCl}_{3}\right) \delta: 167.7$, $138.8,133.1,132.3,132.2,128.7,127.8,126.2\left(\mathrm{q}, J_{\mathrm{C}-\mathrm{F}}=\right.$ $280.5 \mathrm{~Hz}), 124.0,120.1,60.8\left(\mathrm{q}, J_{\mathrm{C}-\mathrm{F}}=27.0 \mathrm{~Hz}\right), 31.6(\mathrm{q}$, $\left.J_{\mathrm{C}-\mathrm{F}}=3.0 \mathrm{~Hz}\right), 21.4 ;{ }^{19} \mathrm{~F}$ NMR $\left(376 \mathrm{MHz}, \mathrm{CDCl}_{3}\right) \delta:-$ $75.46\left(\mathrm{~d}, J_{\mathrm{C}-\mathrm{F}}=3.76 \mathrm{~Hz}\right)$.

$N^{\prime}$-(1-三氟甲基丁-3-烯-1-基)-4-甲基苯甲酰肼(3d): $61.3 \mathrm{mg}$, 白色固体, 产率 $85 \%$. m.p. $100 \sim 101{ }^{\circ} \mathrm{C}$ (文献 值 ${ }^{[15 \mathrm{a}]}$ : $\left.100 \sim 101{ }^{\circ} \mathrm{C}\right) ;{ }^{1} \mathrm{H}$ NMR (600 MHz, $\left.\mathrm{CDCl}_{3}\right) \delta: 7.82$ (s, 1H), 7.62 (d, $J=7.8 \mathrm{~Hz}, 2 \mathrm{H}), 7.23$ (d, $J=10.2 \mathrm{~Hz}, 2 \mathrm{H})$, $6.03 \sim 5.96(\mathrm{~m}, 1 \mathrm{H}), 5.31(\mathrm{~d}, J=10.2 \mathrm{~Hz}, 1 \mathrm{H}), 5.29$ (s, 1H), 4.93 (d, $J=7.8 \mathrm{~Hz}, 1 \mathrm{H}), 3.48$ (s, 1H), 2.60 (d, $J=$ $15.0 \mathrm{~Hz}, 1 \mathrm{H}), 2.39(\mathrm{~s}, 3 \mathrm{H}), 2.37 \sim 2.31(\mathrm{~m}, 1 \mathrm{H}) ;{ }^{13} \mathrm{C} \mathrm{NMR}$ $\left(100 \mathrm{MHz}, \mathrm{CDCl}_{3}\right) \delta: 67.5,142.9,132.2,129.5,129.4$, $127.0,126.2\left(\mathrm{q}, J_{\mathrm{C}-\mathrm{F}}=280.0 \mathrm{~Hz}\right), 120.2,60.7\left(\mathrm{q}, J_{\mathrm{C}-\mathrm{F}}=\right.$ $26.0 \mathrm{~Hz}), 31.6\left(\mathrm{q}, J_{\mathrm{C}-\mathrm{F}}=2.0 \mathrm{~Hz}\right), 21.6 ;{ }^{19} \mathrm{~F}$ NMR $(376$ $\left.\mathrm{MHz}, \mathrm{CDCl}_{3}\right) \delta:-75.68(\mathrm{~d}, J=7.52 \mathrm{~Hz})$.

$N^{\prime}$-(1-三氟甲基丁-3-烯-1-基)-4-甲氧基苯甲酰胇 (3e): $64.9 \mathrm{mg}$, 白色固体, 产率 75\%. m.p. 99 100 ${ }^{\circ} \mathrm{C}$ (文献值 ${ }^{[15 a]}$ : 99 100 $\left.{ }^{\circ} \mathrm{C}\right) ;{ }^{1} \mathrm{H} \mathrm{NMR}\left(600 \mathrm{MHz}, \mathrm{CDCl}_{3}\right) \delta$ : $7.74(\mathrm{~s}, 1 \mathrm{H}), 7.70$ (d, $J=8.4 \mathrm{~Hz}, 2 \mathrm{H}), 6.93(\mathrm{~d}, J=9.0 \mathrm{~Hz}$, $2 \mathrm{H}), 6.03 \sim 5.96(\mathrm{~m}, 1 \mathrm{H}), 5.31(\mathrm{~d}, J=9.0 \mathrm{~Hz}, 1 \mathrm{H}), 5.29(\mathrm{~s}$, $1 \mathrm{H}), 4.93(\mathrm{~d}, J=4.8 \mathrm{~Hz}, 1 \mathrm{H}), 3.85(\mathrm{~s}, 3 \mathrm{H}), 3.51 \sim 3.45(\mathrm{~m}$, $1 \mathrm{H}), 2.60(\mathrm{~d}, J=15.0 \mathrm{~Hz}, 1 \mathrm{H}), 2.37 \sim 2.31(\mathrm{~m}, 1 \mathrm{H}) ;{ }^{13} \mathrm{C}$ NMR (150 MHz, $\left.\mathrm{CDCl}_{3}\right) \delta: 167.2,162.8,132.3,128.9$, $126.2\left(\mathrm{q}, J_{\mathrm{C}-\mathrm{F}}=280.5 \mathrm{~Hz}\right), 124.5,120.0,114.1,60.8(\mathrm{q}$, $\left.J_{\mathrm{C}-\mathrm{F}}=27.0 \mathrm{~Hz}\right), 55.5,31.7\left(\mathrm{q}, J_{\mathrm{C}-\mathrm{F}}=1.5 \mathrm{~Hz}\right) ;{ }^{19} \mathrm{~F}$ NMR $\left(376 \mathrm{MHz}, \mathrm{CDCl}_{3}\right) \delta:-75.47(\mathrm{~d}, J=7.52 \mathrm{~Hz})$.

$N^{\prime}$-(1-三氟甲基丁-3-烯-1-基)-2-氯苯甲酰肼(3f): $38.0 \mathrm{mg}$, 白色固体, 产率 $32 \%$. m.p. $82 \sim 83{ }^{\circ} \mathrm{C} ;{ }^{1} \mathrm{H}$ NMR (600 MHz, $\left.\mathrm{CDCl}_{3}\right) \delta: 7.84(\mathrm{br}, 1 \mathrm{H}), 7.63 \sim 7.61(\mathrm{~m}$, $1 \mathrm{H}), 7.43 \sim 7.38(\mathrm{~m}, 2 \mathrm{H}), 7.35 \sim 7.32(\mathrm{~m}, 1 \mathrm{H}), 6.04 \sim 5.97$ (m, 1H), 5.34 (d, $J=10.2 \mathrm{~Hz}, 2 \mathrm{H}), 5.31(\mathrm{~s}, 1 \mathrm{H}), 4.94(\mathrm{~d}$, $J=6.0 \mathrm{~Hz}, 1 \mathrm{H}), 3.57 \sim 3.52(\mathrm{~m}, 1 \mathrm{H}), 2.63(\mathrm{~d}, J=14.4 \mathrm{~Hz}$, $1 \mathrm{H}), 2.39 \sim 2.34(\mathrm{~m}, 1 \mathrm{H}) ;{ }^{13} \mathrm{C} \mathrm{NMR}\left(150 \mathrm{MHz}, \mathrm{CDCl}_{3}\right) \delta$ : $166.3,132.6,132.2,132.1,131.3,130.6,130.4,127.3$, $126.1\left(\mathrm{q}, J_{\mathrm{C}-\mathrm{F}}=280.5 \mathrm{~Hz}\right), 120.4,60.8\left(\mathrm{q}, J_{\mathrm{C}-\mathrm{F}}=27.0 \mathrm{~Hz}\right)$, 31.6 (q, $\left.J_{\mathrm{C}-\mathrm{F}}=3.0 \mathrm{~Hz}\right) ;{ }^{19} \mathrm{~F}$ NMR $\left(376 \mathrm{MHz}, \mathrm{CDCl}_{3}\right) \delta$ : -75.48 (d, $J=7.52 \mathrm{~Hz}$ ); HRMS (ESI) calcd for $\mathrm{C}_{12} \mathrm{H}_{12} \mathrm{ClF}_{3} \mathrm{~N}_{2} \mathrm{ONa}[\mathrm{M}+\mathrm{Na}]^{+}$315.0482, found 315.0482.

$N^{\prime}$-(1-三氟甲基丁-3-烯-1-基)-3-氯苯甲酰肼 (3g): $97.5 \mathrm{mg}$, 白色固体, 产率 $82 \%$. m.p. $115 \sim 116{ }^{\circ} \mathrm{C} ;{ }^{1} \mathrm{H}$ NMR (600 MHz, $\left.\mathrm{CDCl}_{3}\right) \delta: 7.85$ (br, 1H), 7.73 (t, $J=1.8$ $\mathrm{Hz}, 1 \mathrm{H}), 7.59$ (d, $J=7.8 \mathrm{~Hz}, 1 \mathrm{H}), 7.51(\mathrm{~d}, J=8.4 \mathrm{~Hz}, 1 \mathrm{H})$, $7.38(\mathrm{t}, J=7.8 \mathrm{~Hz}, 1 \mathrm{H}), 6.02 \sim 5.95(\mathrm{~m}, 1 \mathrm{H}), 5.32(\mathrm{~d}, J=$ $9.6 \mathrm{~Hz}, 1 \mathrm{H}), 5.30(\mathrm{~s}, 1 \mathrm{H}), 4.92(\mathrm{~d}, J=6.0 \mathrm{~Hz}, 1 \mathrm{H}), 3.52 \sim$ $3.46(\mathrm{~m}, 1 \mathrm{H}), 2.62(\mathrm{~d}, J=14.4 \mathrm{~Hz}, 1 \mathrm{H}), 2.37 \sim 2.31(\mathrm{~m}$, $1 \mathrm{H}) ;{ }^{13} \mathrm{C}$ NMR $\left(150 \mathrm{MHz}, \mathrm{CDCl}_{3}\right) \delta: 166.3,135.2,134.0$, $132.4,132.1,130.3,127.5,126.1\left(\mathrm{q}, J_{\mathrm{C}-\mathrm{F}}=280.5 \mathrm{~Hz}\right)$, $125.1,120.4,60.7\left(\mathrm{q}, J_{\mathrm{C}-\mathrm{F}}=27.0 \mathrm{~Hz}\right), 31.6\left(\mathrm{q}, J_{\mathrm{C}-\mathrm{F}}=1.5\right.$ $\mathrm{Hz}) ;{ }^{19} \mathrm{~F}$ NMR $\left(376 \mathrm{MHz}, \mathrm{CDCl}_{3}\right) \delta:-75.46(\mathrm{~d}, J=7.52$ $\mathrm{Hz}$ ); HRMS (ESI) calcd for $\mathrm{C}_{12} \mathrm{H}_{12} \mathrm{ClF}_{3} \mathrm{~N}_{2} \mathrm{ONa}[\mathrm{M}+\mathrm{Na}]^{+}$ 315.0482, found 315.0486.

$N^{\prime}$-(1-三氟甲基丁-3-烯-1-基)-4-氯苯甲酰肼(3h): $102.2 \mathrm{mg}$, 白色固体, 产率 $86 \%$. m.p. $114 \sim 115{ }^{\circ} \mathrm{C}$ (文 献值 ${ }^{[15 a]}$ : $\left.114 \sim 115{ }^{\circ} \mathrm{C}\right) ;{ }^{1} \mathrm{H}$ NMR $\left(600 \mathrm{MHz}, \mathrm{CDCl}_{3}\right) \delta$ : 7.74 (br, 1H), 7.67 (d, $J=8.4 \mathrm{~Hz}, 2 \mathrm{H}), 7.42$ (d, $J=8.4 \mathrm{~Hz}$, $2 \mathrm{H}), 6.02 \sim 5.95(\mathrm{~m}, 1 \mathrm{H}), 5.33(\mathrm{~d}, J=7.8 \mathrm{~Hz}, 1 \mathrm{H}), 5.31(\mathrm{~s}$, $1 \mathrm{H}), 4.92(\mathrm{~d}, J=6.0 \mathrm{~Hz}, 1 \mathrm{H}), 3.51 \sim 3.46(\mathrm{~m}, 1 \mathrm{H}), 2.62(\mathrm{~d}$, 
$J=14.4 \mathrm{~Hz}, 1 \mathrm{H}), 2.37 \sim 2.31(\mathrm{~m}, 1 \mathrm{H}) ;{ }^{13} \mathrm{C}$ NMR $(150$ $\left.\mathrm{MHz}, \mathrm{CDCl}_{3}\right) \delta: 166.6,138.7,132.1,130.7,129.2,128.5$, $126.2\left(\mathrm{q}, J_{\mathrm{C}-\mathrm{F}}=280.5 \mathrm{~Hz}\right), 120.3,60.7\left(\mathrm{q}, J_{\mathrm{C}-\mathrm{F}}=27.0 \mathrm{~Hz}\right)$, $31.6\left(\mathrm{q}, J_{\mathrm{C}-\mathrm{F}}=3.0 \mathrm{~Hz}\right) ;{ }^{19} \mathrm{~F}$ NMR $\left(376 \mathrm{MHz}, \mathrm{CDCl}_{3}\right) \delta$ : $-75.46(\mathrm{~d}, J=7.52 \mathrm{~Hz})$.

$N^{\prime}$-(1-三氟甲基丁-3-烯-1-基)-4-氟苯甲酰肼 (3i): $67.1 \mathrm{mg}$, 白色固体, 产率 $81 \%$. m.p. $81 \sim 82{ }^{\circ} \mathrm{C}$ (文献 值 $\left.{ }^{[15 a]}: 81 \sim 82{ }^{\circ} \mathrm{C}\right) ;{ }^{1} \mathrm{H}$ NMR $\left(600 \mathrm{MHz}, \mathrm{CDCl}_{3}\right) \delta: 7.75 \sim$ $7.74(\mathrm{~m}, 3 \mathrm{H}), 7.14(\mathrm{t}, J=8.4 \mathrm{~Hz}, 2 \mathrm{H}), 6.02 \sim 5.96(\mathrm{~m}, 1 \mathrm{H})$, $5.33(\mathrm{~d}, J=8.4 \mathrm{~Hz}, 1 \mathrm{H}), 5.31(\mathrm{~s}, 1 \mathrm{H}), 4.92(\mathrm{~d}, J=6.0 \mathrm{~Hz}$, $1 \mathrm{H}), 3.51 \sim 3.46(\mathrm{~m}, 1 \mathrm{H}), 2.62(\mathrm{~d}, J=15.0 \mathrm{~Hz}, 1 \mathrm{H}), 2.37 \sim$ $2.31(\mathrm{~m}, 1 \mathrm{H}) ;{ }^{13} \mathrm{C}$ NMR $\left(150 \mathrm{MHz}, \mathrm{CDCl}_{3}\right) \delta: 166.6,165.3$ $\left(\mathrm{d}, J_{\mathrm{C}-\mathrm{F}}=252.0 \mathrm{~Hz}\right), 132.1,129.5\left(\mathrm{~d}, J_{\mathrm{C}-\mathrm{F}}=9.0 \mathrm{~Hz}\right), 128.5$ $\left(\mathrm{d}, J_{\mathrm{C}-\mathrm{F}}=3.0 \mathrm{~Hz}\right), 126.2\left(\mathrm{q}, J_{\mathrm{C}-\mathrm{F}}=279.0 \mathrm{~Hz}\right), 120.3,116.1$ $\left(\mathrm{d}, J_{\mathrm{C}-\mathrm{F}}=21.0 \mathrm{~Hz}\right), 60.8\left(\mathrm{q}, J_{\mathrm{C}-\mathrm{F}}=27.0 \mathrm{~Hz}\right), 31.6\left(\mathrm{q}, J_{\mathrm{C}-\mathrm{F}}=\right.$ $3.0 \mathrm{~Hz}) ;{ }^{19} \mathrm{~F}$ NMR $\left(376 \mathrm{MHz}, \mathrm{CDCl}_{3}\right) \delta$ : $-75.49(\mathrm{~d}, J=$ $7.52 \mathrm{~Hz}),-107.10 \sim-107.17(\mathrm{~m})$.

$N^{\prime}$-(1-三氟甲基丁-3-烯-1-基)-3-溴苯甲酰肼 (3j): $72.8 \mathrm{mg}$, 白色固体, 产率 $72 \%$. m.p. $80 \sim 82{ }^{\circ} \mathrm{C}$ (文献 值 $\left.{ }^{[15 a]}: 80 \sim 82{ }^{\circ} \mathrm{C}\right) ;{ }^{1} \mathrm{H}$ NMR $\left(600 \mathrm{MHz}, \mathrm{CDCl}_{3}\right) \delta: 7.88$ $(\mathrm{t}, J=1.2 \mathrm{~Hz}, 1 \mathrm{H}), 7.86(\mathrm{~d}, J=6.0 \mathrm{~Hz}, 1 \mathrm{H}), 7.67 \sim 7.63(\mathrm{~m}$, $2 \mathrm{H}), 7.32(\mathrm{t}, J=7.8 \mathrm{~Hz}, 1 \mathrm{H}), 6.01 \sim 5.95(\mathrm{~m}, 1 \mathrm{H}), 5.32(\mathrm{~d}$, $J=9.0 \mathrm{~Hz}, 1 \mathrm{H}), 5.30(\mathrm{~s}, 1 \mathrm{H}), 4.91(\mathrm{~d}, J=6.0 \mathrm{~Hz}, 1 \mathrm{H})$, $3.51 \sim 3.46(\mathrm{~m}, 1 \mathrm{H}), 2.62(\mathrm{~d}, J=13.2 \mathrm{~Hz}, 1 \mathrm{H}), 2.37 \sim 2.31$ $(\mathrm{m}, 1 \mathrm{H}) ;{ }^{13} \mathrm{C}$ NMR $\left(150 \mathrm{MHz}, \mathrm{CDCl}_{3}\right) \delta: 166.2,135.3$, $134.2,132.1,130.5,130.4,126.1\left(\mathrm{q}, J_{\mathrm{C}-\mathrm{F}}=279.0 \mathrm{~Hz}\right)$, $125.5,123.1,120.3,60.7\left(\mathrm{q}, J_{\mathrm{C}-\mathrm{F}}=27.0 \mathrm{~Hz}\right), 31.6(\mathrm{q}$, $\left.J_{\mathrm{C}-\mathrm{F}}=1.5 \mathrm{~Hz}\right) ;{ }^{19} \mathrm{~F}$ NMR $\left(376 \mathrm{MHz}, \mathrm{CDCl}_{3}\right) \delta:-75.44(\mathrm{~d}$, $J=7.52 \mathrm{~Hz})$.

$N^{\prime}$-(1-三氟甲基丁-3-烯-1-基)-4-溴苯甲酰肼 (3k): $80.9 \mathrm{mg}$, 白色固体, 产率 $80 \%$. m.p. $130 \sim 131{ }^{\circ} \mathrm{C} ;{ }^{1} \mathrm{H}$ NMR (600 MHz, $\left.\mathrm{CDCl}_{3}\right) \delta: 7.92(\mathrm{br}, 1 \mathrm{H}), 7.88$ (t, $J=1.2$ $\mathrm{Hz}, 1 \mathrm{H}), 7.66 \sim 7.63(\mathrm{~m}, 2 \mathrm{H}), 7.31(\mathrm{t}, J=7.8 \mathrm{~Hz}, 1 \mathrm{H})$, $6.01 \sim 5.94(\mathrm{~m}, 1 \mathrm{H}), 5.33(\mathrm{~d}, J=1.2 \mathrm{~Hz}, 1 \mathrm{H}), 5.31 \sim 5.29$ $(\mathrm{m}, 1 \mathrm{H}), 4.92(\mathrm{~d}, J=6.6 \mathrm{~Hz}, 1 \mathrm{H}), 3.52 \sim 3.46(\mathrm{~m}, 1 \mathrm{H})$, $2.63 \sim 2.59(\mathrm{~m}, 1 \mathrm{H}), 2.37 \sim 2.31(\mathrm{~m}, 1 \mathrm{H}) ;{ }^{13} \mathrm{C}$ NMR $(150$ $\left.\mathrm{MHz}, \mathrm{CDCl}_{3}\right) \delta: 166.2,135.3,134.2,132.1,130.5,130.4$, $125.6,126.3\left(\mathrm{q}, J_{\mathrm{C}-\mathrm{F}}=279.0 \mathrm{~Hz}\right), 123.1,120.3,60.7(\mathrm{q}$, $\left.J_{\mathrm{C}-\mathrm{F}}=27.0 \mathrm{~Hz}\right), 31.6\left(\mathrm{q}, J_{\mathrm{C}-\mathrm{F}}=1.5 \mathrm{~Hz}\right) ;{ }^{19} \mathrm{~F}$ NMR $(376$ $\left.\mathrm{MHz}, \mathrm{CDCl}_{3}\right) \delta:-75.46(\mathrm{~d}, J=7.52 \mathrm{~Hz}$ ); HRMS (ESI) calcd for $\mathrm{C}_{12} \mathrm{H}_{12} \mathrm{Br}_{2} \mathrm{~F}_{3} \mathrm{~N}_{2} \mathrm{ONa}[\mathrm{M}+\mathrm{Na}]^{+}$358.9977, found 358.9976.

$N^{\prime}$-(1-三氟甲基丁-3-烯-1-基)-4-三氟甲基苯甲酰肼 (3I): $61.7 \mathrm{mg}$, 白色固体, 产率 $63 \%$. m.p. $87 \sim 89{ }^{\circ} \mathrm{C}$ (文 献值 $\left.{ }^{[15 a]}: 87 \sim 89{ }^{\circ} \mathrm{C}\right) ;{ }^{1} \mathrm{H}$ NMR $\left(600 \mathrm{MHz}, \mathrm{CDCl}_{3}\right) \delta: 7.96$ (s, 1H), $7.84(\mathrm{~d}, J=7.8 \mathrm{~Hz}, 2 \mathrm{H}), 7.71(\mathrm{~d}, J=8.4 \mathrm{~Hz}, 2 \mathrm{H})$, $6.02 \sim 5.95(\mathrm{~m}, 1 \mathrm{H}), 5.33(\mathrm{~d}, J=10.2 \mathrm{~Hz}, 1 \mathrm{H}), 5.31(\mathrm{~s}$, $1 \mathrm{H}), 4.95(\mathrm{~d}, J=6.0 \mathrm{~Hz}, 1 \mathrm{H}), 3.54 \sim 3.48(\mathrm{~m}, 1 \mathrm{H}), 2.63(\mathrm{~d}$, $J=14.4 \mathrm{~Hz}, 1 \mathrm{H}), 2.37 \sim 2.31(\mathrm{~m}, 1 \mathrm{H}) ;{ }^{13} \mathrm{C}$ NMR $(150$ $\left.\mathrm{MHz}, \mathrm{CDCl}_{3}\right) \delta: 166.3,135.6,134.1\left(\mathrm{q}, J_{\mathrm{C}-\mathrm{F}}=31.5 \mathrm{~Hz}\right)$, $132.0,127.6,126.1\left(\mathrm{q}, J_{\mathrm{C}-\mathrm{F}}=280.5 \mathrm{~Hz}\right), 126.0\left(\mathrm{q}, J_{\mathrm{C}-\mathrm{F}}=\right.$ $4.5 \mathrm{~Hz}), 123.6\left(\mathrm{q}, J_{\mathrm{C}-\mathrm{F}}=270.0 \mathrm{~Hz}\right), 120.4,60.7\left(\mathrm{q}, J_{\mathrm{C}-\mathrm{F}}=\right.$ $27.0 \mathrm{~Hz}), 31.6\left(\mathrm{q}, J_{\mathrm{C}-\mathrm{F}}=3.0 \mathrm{~Hz}\right) ;{ }^{19} \mathrm{~F}$ NMR $(376 \mathrm{MHz}$, $\left.\mathrm{CDCl}_{3}\right) \delta:-63.58(\mathrm{~d}, J=3.76 \mathrm{~Hz}),-75.49$ (d, $J=3.76$ $\mathrm{Hz})$.

$N^{\prime}$-(1-三氟甲基丁-3-烯-1-基)-2-菜甲酰肼(3m): 77.7 $\mathrm{mg}$, 白色固体, 产率 $84 \%$. m.p. $108 \sim 109{ }^{\circ} \mathrm{C} ;{ }^{1} \mathrm{H}$ NMR $\left(600 \mathrm{MHz}, \mathrm{CDCl}_{3}\right) \delta: 8.23(\mathrm{~s}, 1 \mathrm{H}), 8.05(\mathrm{~d}, J=5.4 \mathrm{~Hz}$, $1 \mathrm{H}), 7.87 \sim 7.84(\mathrm{~m}, 3 \mathrm{H}), 7.76 \sim 7.74(\mathrm{~m}, 1 \mathrm{H}), 7.58 \sim 7.51$ (m, $2 \mathrm{H}), 6.05 \sim 5.98(\mathrm{~m}, 1 \mathrm{H}), 5.32(\mathrm{~d}, J=10.2 \mathrm{~Hz}, 1 \mathrm{H})$, $5.30(\mathrm{~s}, 1 \mathrm{H}), 5.01(\mathrm{~d}, J=6.0 \mathrm{~Hz}, 1 \mathrm{H}), 3.57 \sim 3.52(\mathrm{~m}, 1 \mathrm{H})$, $2.63(\mathrm{~d}, J=15.0 \mathrm{~Hz}, 1 \mathrm{H}), 2.39 \sim 2.34(\mathrm{~m}, 1 \mathrm{H}) ;{ }^{13} \mathrm{C} \mathrm{NMR}$ $\left(100 \mathrm{MHz}, \mathrm{CDCl}_{3}\right) \delta: 167.7,135.1,132.6,132.2,129.4$, $129.1,128.8,128.1,127.9,127.8,127.1,126.2\left(\mathrm{q}, J_{\mathrm{C}-\mathrm{F}}=\right.$ $279.0 \mathrm{~Hz}), 123.2,120.3,60.8\left(\mathrm{q}, J_{\mathrm{C}-\mathrm{F}}=27.0 \mathrm{~Hz}\right), 31.7$ (q, $\left.J_{\mathrm{C}-\mathrm{F}}=2.0 \mathrm{~Hz}\right) ;{ }^{19} \mathrm{~F}$ NMR $\left(376 \mathrm{MHz}, \mathrm{CDCl}_{3}\right) \delta:-75.36(\mathrm{~d}$, $J=3.76 \mathrm{~Hz}$ ); HRMS (ESI) calcd for $\mathrm{C}_{16} \mathrm{H}_{15} \mathrm{~F}_{3} \mathrm{~N}_{2} \mathrm{ONa}[\mathrm{M}+$ $\mathrm{Na}]^{+}$331.1029, found 331.1031.

$N^{\prime}$-(1-三氟甲基丁-3-烯-1-基)-2-呋喃甲酰肼 (3n): $64.0 \mathrm{mg}$, 白色固体, 产率 86\%. m.p. $65 \sim 67{ }^{\circ} \mathrm{C}$ (文献 值 ${ }^{[15 a]}$ : $\left.65 \sim 67{ }^{\circ} \mathrm{C}\right) ;{ }^{1} \mathrm{H}$ NMR $\left(600 \mathrm{MHz}, \mathrm{CDCl}_{3}\right) \delta 7.87(\mathrm{~d}$, $J=5.4 \mathrm{~Hz}, 1 \mathrm{H}), 7.47 \sim 7.46(\mathrm{~m}, 1 \mathrm{H}), 7.15(\mathrm{~d}, J=3.6 \mathrm{~Hz}$, $1 \mathrm{H}), 6.52(\mathrm{dd}, J=3.6 \mathrm{~Hz}, 1.8 \mathrm{~Hz}, 1 \mathrm{H}), 6.03 \sim 5.96(\mathrm{~m}$, $1 \mathrm{H}), 5.32(\mathrm{~d}, J=12.0 \mathrm{~Hz}, 1 \mathrm{H}), 5.30(\mathrm{~s}, 1 \mathrm{H}), 4.81(\mathrm{~d}, J=$ $6.6 \mathrm{~Hz}, 1 \mathrm{H}), 3.51 \sim 3.45(\mathrm{~m}, 1 \mathrm{H}), 2.61(\mathrm{~d}, J=15.0 \mathrm{~Hz}$, $1 \mathrm{H}), 2.37 \sim 2.31(\mathrm{~m}, 1 \mathrm{H}) ;{ }^{13} \mathrm{C} \mathrm{NMR}\left(150 \mathrm{MHz}, \mathrm{CDCl}_{3}\right) \delta$ : $158.3,146.3,144.7,132.1,126.1\left(\mathrm{q}, J_{\mathrm{C}-\mathrm{F}}=279.0 \mathrm{~Hz}\right)$, $120.2,115.5,112.3,60.9\left(\mathrm{q}, J_{\mathrm{C}-\mathrm{F}}=27.0 \mathrm{~Hz}\right), 31.6\left(\mathrm{q}, J_{\mathrm{C}-\mathrm{F}}=\right.$ $1.5 \mathrm{~Hz}) ;{ }^{19} \mathrm{~F}$ NMR $\left(376 \mathrm{MHz}, \mathrm{CDCl}_{3}\right) \delta:-75.68(\mathrm{~d}, J=$ $7.52 \mathrm{~Hz})$.

$N^{\prime}$-(1-三氟甲基丁-3-烯-1-基)-2-噻吩甲酰肼 (30): $66.6 \mathrm{mg}$, 白色固体, 产率 $84 \%$. m.p. $87 \sim 88{ }^{\circ} \mathrm{C} ;{ }^{1} \mathrm{H}$ NMR $\left(600 \mathrm{MHz}, \mathrm{CDCl}_{3}\right) \delta: 7.61(\mathrm{~s}, 1 \mathrm{H}), 7.44 \sim 7.52(\mathrm{~m}$, $2 \mathrm{H}), 7.12 \sim 7.09(\mathrm{~m}, 1 \mathrm{H}), 6.03 \sim 5.96(\mathrm{~m}, 1 \mathrm{H}), 5.32(\mathrm{~d}, J=$ $9.6 \mathrm{~Hz}, 1 \mathrm{H}), 5.30(\mathrm{~s}, 1 \mathrm{H}), 4.85(\mathrm{~d}, J=5.4 \mathrm{~Hz}, 1 \mathrm{H}), 3.54 \sim$ $3.48(\mathrm{~m}, 1 \mathrm{H}), 2.61(\mathrm{~d}, J=15.0 \mathrm{~Hz}, 1 \mathrm{H}), 2.36 \sim 2.30(\mathrm{~m}$, $1 \mathrm{H}) ;{ }^{13} \mathrm{C}$ NMR $\left(150 \mathrm{MHz}, \mathrm{CDCl}_{3}\right) \delta: 162.2,135.9,132.2$, $131.0,128.9,128.0,126.2\left(\mathrm{q}, J_{\mathrm{C}-\mathrm{F}}=280.5 \mathrm{~Hz}\right), 120.3,60.8$ $\left(\mathrm{q}, J_{\mathrm{C}-\mathrm{F}}=27.0 \mathrm{~Hz}\right), 31.6\left(\mathrm{q}, J_{\mathrm{C}-\mathrm{F}}=3.0 \mathrm{~Hz}\right) ;{ }^{19} \mathrm{~F}$ NMR $(376$ $\left.\mathrm{MHz}, \mathrm{CDCl}_{3}\right) \delta$ : $-75.61(\mathrm{~d}, J=7.52 \mathrm{~Hz}$ ); HRMS (ESI) 
calcd for $\mathrm{C}_{10} \mathrm{H}_{11} \mathrm{~F}_{3} \mathrm{~N}_{2} \mathrm{ONaS}[\mathrm{M}+\mathrm{Na}]^{+}$287.0436, found 287.0431.

$N^{\prime}$-(1-三氟甲基丁-3-烯-1-基)-2-噻吩并 [3,2- $b$ ]塞吩 甲酰肼(3p): $54.8 \mathrm{mg}$, 白色固体, 产率 57\%. m.p. 102 $103{ }^{\circ} \mathrm{C} ;{ }^{1} \mathrm{H}$ NMR $\left(600 \mathrm{MHz}, \mathrm{CDCl}_{3}\right) \delta: 7.79 \sim 7.65(\mathrm{~m}$, $2 \mathrm{H}), 7.57 \sim 7.55(\mathrm{~m}, 1 \mathrm{H}), 7.29 \sim 7.27(\mathrm{~m}, 1 \mathrm{H}), 6.03 \sim 5.96$ $(\mathrm{m}, 1 \mathrm{H}), 5.33(\mathrm{~d}, J=7.8 \mathrm{~Hz}, 1 \mathrm{H}), 5.31(\mathrm{~s}, 1 \mathrm{H}), 4.89(\mathrm{~s}$, $1 \mathrm{H}), 3.56 \sim 3.50(\mathrm{~m}, 1 \mathrm{H}), 2.62(\mathrm{~d}, J=13.2 \mathrm{~Hz}, 1 \mathrm{H}), 2.37 \sim$ $2.31(\mathrm{~m}, 1 \mathrm{H}) ;{ }^{13} \mathrm{C}$ NMR $\left(150 \mathrm{MHz}, \mathrm{CDCl}_{3}\right) \delta: 162.7$, $142.6,139.1,136.9,132.1,131.4,126.1$ (q, $J_{\mathrm{C}-\mathrm{F}}=279.0$ $\mathrm{Hz}), 121.5,120.2,119.8,60.7$ (q, $\left.J_{\mathrm{C}-\mathrm{F}}=27.0 \mathrm{~Hz}\right), 31.6 ;{ }^{19} \mathrm{~F}$ NMR $\left(376 \mathrm{MHz}, \mathrm{CDCl}_{3}\right) \delta:-75.39(\mathrm{~d}, J=7.52 \mathrm{~Hz})$; HRMS (ESI) calcd for $\mathrm{C}_{12} \mathrm{H}_{11} \mathrm{~F}_{3} \mathrm{~N}_{2} \mathrm{ONaS}_{2}[\mathrm{M}+\mathrm{Na}]^{+}$ 343.0157, found 343.0154 .

$N^{\prime}$-(1-三氟甲基丁-3-烯-1-基)-环已烷甲酰肼(3q): $57.1 \mathrm{mg}$, 白色固体, 产率 $72 \%$. m.p. 59 61 ${ }^{\circ} \mathrm{C}$ (文献 值 $\left.{ }^{[22]}: 59 \sim 61{ }^{\circ} \mathrm{C}\right) ;{ }^{1} \mathrm{H}$ NMR $\left(600 \mathrm{MHz}, \mathrm{CDCl}_{3}\right) \delta: 7.20(\mathrm{~d}$, $J=6.6 \mathrm{~Hz}, 1 \mathrm{H}), 5.98 \sim 5.91(\mathrm{~m}, 1 \mathrm{H}), 5.28(\mathrm{~d}, J=7.8 \mathrm{~Hz}$, $1 \mathrm{H}), 5.26$ (s, $1 \mathrm{H}), 4.67(\mathrm{~d}, J=6.0 \mathrm{~Hz}, 1 \mathrm{H}), 3.37 \sim 3.31(\mathrm{~m}$, $1 \mathrm{H}), 2.55(\mathrm{~d}, J=14.4 \mathrm{~Hz}, 1 \mathrm{H}), 2.29 \sim 2.24(\mathrm{~m}, 1 \mathrm{H}), 2.07 \sim$ $2.03(\mathrm{~m}, 1 \mathrm{H}) .1 .82 \sim 1.78(\mathrm{~m}, 4 \mathrm{H}), 1.69 \sim 1.67(\mathrm{~m}, 1 \mathrm{H})$, $1.48 \sim 1.41(\mathrm{~m}, 2 \mathrm{H}), 1.28 \sim 1.20(\mathrm{~m}, 3 \mathrm{H}) ;{ }^{13} \mathrm{C}$ NMR $(150$ $\left.\mathrm{MHz}, \mathrm{CDCl}_{3}\right) \delta: 175.8,132.2,126.2\left(\mathrm{~d}, J_{\mathrm{C}-\mathrm{F}}=279.0 \mathrm{~Hz}\right)$, $120.1,60.6\left(\mathrm{q}, J_{\mathrm{C}-\mathrm{F}}=27.0 \mathrm{~Hz}\right), 43.4,31.6\left(\mathrm{q}, J_{\mathrm{C}-\mathrm{F}}=3.0\right.$ $\mathrm{Hz}), 29.4,29.2,25.7,25.7,25.6 ;{ }^{19} \mathrm{~F}$ NMR $(376 \mathrm{MHz}$, $\left.\mathrm{CDCl}_{3}\right) \delta:-76.37(\mathrm{~d}, J=7.52 \mathrm{~Hz})$.

2-苯基- $N^{\prime}$-(1-三氟甲基丁-3-烯-1-基)-乙酰肼(3r): $66.1 \mathrm{mg}$, 白色固体, 产率 $81 \%$. m.p. 96 97 ${ }^{\circ} \mathrm{C}$ (文献 值 ${ }^{[22]}$ : 96 97 $\left.{ }^{\circ} \mathrm{C}\right) ;{ }^{1} \mathrm{H}$ NMR $\left(600 \mathrm{MHz}, \mathrm{CDCl}_{3}\right) \delta: 7.34(\mathrm{t}$, $J=7.2 \mathrm{~Hz}, 2 \mathrm{H}), 7.29$ (t, $J=7.2 \mathrm{~Hz}, 1 \mathrm{H}), 7.26 \sim 7.15(\mathrm{~m}$, $3 \mathrm{H}), 5.93 \sim 5.86(\mathrm{~m}, 1 \mathrm{H}), 5.23(\mathrm{~d}, J=6.0 \mathrm{~Hz}, 1 \mathrm{H}), 5.21(\mathrm{~s}$, $1 \mathrm{H}), 4.65(\mathrm{~d}, J=6.0 \mathrm{~Hz}, 1 \mathrm{H}), 3.52(\mathrm{~s}, 2 \mathrm{H}), 3.39 \sim 3.33(\mathrm{~m}$, $1 \mathrm{H}), 2.52(\mathrm{~d}, J=15.0 \mathrm{~Hz}, 1 \mathrm{H}), 2.26 \sim 2.20(\mathrm{~m}, 1 \mathrm{H}) ;{ }^{13} \mathrm{C}$ NMR $\left(100 \mathrm{MHz}, \mathrm{CDCl}_{3}\right) \delta: 170.7,133.8,132.1,129.3$, $129.1,127.6,126.0\left(\mathrm{~d}, J_{\mathrm{C}-\mathrm{F}}=279.0 \mathrm{~Hz}\right), 120.1,60.4(\mathrm{q}$, $\left.J_{\mathrm{C}-\mathrm{F}}=27.0 \mathrm{~Hz}\right), 41.8,31.5\left(\mathrm{q}, J_{\mathrm{C}-\mathrm{F}}=3.0 \mathrm{~Hz}\right) ;{ }^{19} \mathrm{~F} \mathrm{NMR}$ $\left(376 \mathrm{MHz}, \mathrm{CDCl}_{3}\right) \delta:-75.58(\mathrm{~d}, J=7.52 \mathrm{~Hz})$.

2-甲基- $N^{\prime}$-(1-三氟甲基丁-3-烯-1-基)-丙酰肼(3s): $21.5 \mathrm{mg}$, 白色固体, 产率 $32 \%$. m.p. $76 \sim 77{ }^{\circ} \mathrm{C} ;{ }^{1} \mathrm{H}$ NMR (600 MHz, $\left.\mathrm{CDCl}_{3}\right) \delta: 7.20(\mathrm{~s}, 1 \mathrm{H}), 5.98 \sim 5.91(\mathrm{~m}$, $1 \mathrm{H}), 5.28(\mathrm{~d}, J=7.8 \mathrm{~Hz}, 1 \mathrm{H}), 5.26(\mathrm{~s}, 1 \mathrm{H}), 4.66$ (d, $J=6.6$ $\mathrm{Hz}, 1 \mathrm{H}), 3.38 \sim 3.24(\mathrm{~m}, 1 \mathrm{H}), 2.56(\mathrm{~d}, J=13.8 \mathrm{~Hz}, 1 \mathrm{H})$, $2.35 \sim 2.24(\mathrm{~m}, 2 \mathrm{H}), 1.16(\mathrm{~d}, J=6.0 \mathrm{~Hz}, 3 \mathrm{H}), 1.15$ (d, $J=$ $6.0 \mathrm{~Hz}, 3 \mathrm{H}) ;{ }^{13} \mathrm{C}$ NMR (150 MHz, $\left.\mathrm{CDCl}_{3}\right) \delta: 176.7,132.2$, $126.2(\mathrm{~d}, J=279.0 \mathrm{~Hz}), 120.1,60.5\left(\mathrm{q}, J_{\mathrm{C}-\mathrm{F}}=27.0 \mathrm{~Hz}\right)$,
33.6, $31.6\left(\mathrm{q}, J_{\mathrm{C}-\mathrm{F}}=1.5 \mathrm{~Hz}\right), 19.4,19.1 ;{ }^{19} \mathrm{~F}$ NMR $(376$ $\left.\mathrm{MHz}, \mathrm{CDCl}_{3}\right) \delta:-75.62(\mathrm{~d}, J=7.52 \mathrm{~Hz}$ ); HRMS (ESI) calcd for $\mathrm{C}_{9} \mathrm{H}_{15} \mathrm{~F}_{3} \mathrm{~N}_{2} \mathrm{ONa}[\mathrm{M}+\mathrm{Na}]^{+} 247.1029$, found 247.1028 .

3.2.2 三氟甲基酰腙与烯丙基硼酸频哪醇酯的烯丙 基化反应

在 $50 \mathrm{~mL}$ 的圆底烧瓶中, 依次加入三氟甲基酰腙 $\mathbf{1 a}$ $(0.065 \mathrm{~g}, 0.3 \mathrm{mmol})$ 、三氟甲磺酸铟 $(0.034 \mathrm{~g}, 0.06 \mathrm{mmol})$ 、 烯丙基硼酸频哪醇酯 4 (0.076 g, $0.45 \mathrm{mmol})$ 和乙腈(3 $\mathrm{mL}$ ). 将此混合物回流状态下反应 $3 \mathrm{~h}, \mathrm{TLC}$ 检测反应完 全. 向反应体系中加入饱和氯化钠溶液 $(5 \mathrm{~mL})$ 淬灭反应, 然后用乙酸乙酯萃取 $(10 \mathrm{~mL} \times 3)$, 分出有机相, 经无水 硫酸镁干燥, 除去溶剂后进行柱层析 $[V$ (石油醚) : $V($ 乙 酸乙酯 $)=5: 1$ 分离得产物 3a $(69.7 \mathrm{mg})$, 白色固体, 产 率 $90 \%$. 化合物 $\mathbf{3 d} \sim \mathbf{3 e}, \mathbf{3 h} \sim \mathbf{3 p}, \mathbf{3 r}, \mathbf{3 s}$ 的合成方法同 $\mathbf{3 a}$.

辅助材料(Supporting Information) 产物 3a $\sim 3 \mathrm{~s}$ 的 ${ }^{1} \mathrm{H}$ $\mathrm{NMR},{ }^{13} \mathrm{C}$ NMR 和 ${ }^{19} \mathrm{~F}$ NMR 以及高分辨质谱谱图. 这些 材料可以免费从本刊网站(http://sioc-journal.cn/)上下载.

\section{References}

[1] For reviews, see: (a) Sugiura, M.; Kobayashi, S. Angew. Chem., Int. Ed. 2005, 44, 5176.

(b) Friestad, G. K. Eur. J. Org. Chem. 2005, 2005, 3157.

[2] For selected examples, see: (a) Wu, P.-L.; Peng, S.-Y.; Magrath, J. Synthesis 1995, 435.

(b) Burk, M. J.; Feaster, J. E. J. Am. Chem. Soc. 1992, 114, 6266.

(c) Meng, J.; Wen, M.; Zhang, S.; Pan, P.; Yu, X.; Deng, W.-P. J. Org. Chem. 2017, 82, 1676.

(d) Kong, Y.; Tang, M.; Wang, Y. Org. Lett. 2014, 16, 576.

(e) Liu, Y.-L.; Yin, X.-P.; Zhou, J. Chin. J. Chem. 2018, 36, 321.

(f) Zamfir, A.; Tsogoeva, S. B. Org. Lett. 2010, 12, 188.

(g) Duan, X.-Y.; Zhou, N.-N.; Fang, R.; Yang, X.-L.; Yu, W.; Han, B. Angew. Chem., Int. Ed. 2014, 53, 3158.

[3] (a) Chauhan, J.; Ravva, M. K.; Sen, S. Org. Lett. 2019, 21, 6562. (b) Wang, Y.; Wang, K.-H.; Su, Y.; Yang, Z.; Wen, L.; Liu, L.; Wang, J.; Huang, D.; Hu, Y. J. Org. Chem. 2018, 83, 939. (c) Tokumaru, K.; Johnston, J. N. Chem. Sci. 2017, 8, 3187.

[4] (a) Mishra, M.; Tiwari, K.; Mourya, P.; Singh, M. M.; Singh, V. P. Polyhedron 2015, 89, 29.

(b) Lian, S.; Su, H.; Zhao, B.-X.; Liu, W.-Y.; Zheng, L.-W.; Miao, J.-Y. Bioorg. Med. Chem. 2009, 17, 7085.

(c) Toraskar, M. P.; Kadam, V. J.; Kulkarni, V. M. Int. J. ChemTech Res. 2009, 1, 1194.

(d) Wheelock, C. E.; Nakagawa, Y.; Harada, T.; Oikawa, N.; Akamatsu, M.; Smagghe, G.; Stefanou, D.; Iatrou, K.; Swevers, L. Bioorg. Med. Chem. 2006, 14, 1143.

[5] (a) Yoneya, M.; Takada, S.; Maeda, Y.; Yokoyama, H. Liq. Cryst. 2008, 35, 339.

(b) Cui, H.; Xu, Y.; Zhang, Z.-F. Anal. Chem. 2004, 76, 4002.

[6] (a) Yus, M.; González-Gómez, J. C.; Foubelo, F. Chem. Rev. 2013, $113,5595$.

(b) Kobayashi, S.; Hamada, T.; Manabe, K. Synlett 2001, 1140.

[7] (a) Tan, K. L.; Jacobsen, E. N. Angew. Chem., Int. Ed. 2007, 46, 1315 .

(b) Kargbo, R.; Takahashi, Y.; Bhor, S.; Cook, G. R.; Lloyd-Jones, G. C.; Shepperson, I. R. J. Am. Chem. Soc. 2007, 129, 3846. 
[8] (a) Schneider, U.; Chen, I.-H.; Kobayashi, S. Org. Lett. 2008, 10, 737.

(b) Chakrabarti, A.; Konishi, H.; Yamaguchi, M.; Schneider, U.; Kobayashi, S. Angew. Chem., Int. Ed. 2010, 49, 1838.

[9] (a) Lade, J. J.; Pardeshi, S. D.; Vadagaonkar, K. S.; Murugan, K.; Chaskar, A. C. RSC Adv. 2017, 7, 8011.

(b) Friestad, G. K.; Korapala, C. S.; Ding, H. J. Org. Chem. 2006, $71,281$.

[10] Zhu, Y.; Han, J.; Wang, J.; Shibata, N.; Sodeoka, M.; Soloshonok, V. A.; Coelho, J. S.; Toste, F. D. Chem. Rev. 2018, 118, 3887.

[11] (a) Wang, J.; Sánchez-Roselló, M.; Aceña, J. L.; del Pozo, C.; Sorochinsky, A. E.; Fustero, S.; Soloshonok, V. A.; Liu, H. Chem. Rev. 2014, 114, 2432.

(b) Hagmann, W. K. J. Med. Chem. 2008, 51, 4359.

(c) Müller, K.; Faeh, C.; Diederich, F. Science 2007, 317, 1881.

(d) Böhm, H.-J.; Banner, D.; Bendels, S.; Kansy, M.; Kuhn, B.; Müller, K.; Obst-Sander, U.; Stahl, M. ChemBioChem 2004, 5, 637.

[12] For selected reviews, see: (a) Alonso, C.; Marigorta, E. M.; Rubiales, G.; Palacios, F. Chem. Rev. 2015, 115, 1847.

(b) Liang, T.; Neumann, C. N.; Ritter, T. Angew. Chem., Int. Ed. 2013, 52, 8214 .

(c) Qing, F. Chin. J. Org. Chem. 2012, 32, 815 (in Chinese). (卿凤悀, 有机化学, 2012, 32, 815.)

[13] For recent articles, see: (a) Trost, B. M.; Gholami, H.; Zell, D. J. Am. Chem. Soc. 2019, 141, 11446.

(b) Ji, X.; Shi, G.; Zhang, Y. Chin. J. Org. Chem. 2019, 39, 929 (in Chinese).

(季小明，史广法，张扬会，有机化学, 2019, 39, 929.)

(c) Yoritate, M.; Londregan, A. T.; Lian, Y.; Hartwig, J. F. J. Org. Chem. 2019, 84, 15767

(d) Liu, Y.-L.; Yin, X.-P.; Zhou, J. Chin. J. Chem. 2018, 36, 321.

[14] (a) Moschner, J.; Stulberg, V.; Fernandes, R.; Huhmann, S.; Leppkes, J.; Koksch, B. Chem. Rev. 2019, 119, 10718.

(b) Fustero, S.; Simón-Fuentes, A.; Barrio, P.; Haufe, G. Chem. Rev. 2015, 115,871 .

(c) Liu, J.; Huang, D.; Wang, X.; Zong, W.; Su, Y.; Wang, K.; Hu, Y. Chin. J. Org. Chem. 2019, 39, 1767 (in Chinese).

(刘佳欣, 黄丹凤, 王小平, 宗吴中, 苏瀛鹏, 王克虎, 胡雨来. 有机化学, 2019, 39, 1767.)

(d) Wang, J.; Li, F.; Xu, Y.; Wang, J.; Wu, Z.; Yang, C.; Liu, L. Chin. J. Org. Chem. 2018, 38, 1155 (in Chinese).

(王晶晶, 李峰, 徐妍, 王娟, 武紫燕, 杨成玉, 刘澜涛, 有机化 学, 2018, 38, 1155.)

(e) Zhang, F.-G.; Lv, N.; Zheng, Y.; Ma, J.-A. Chin. J. Chem. 2018,
36,723 .

[15] (a) Li, J.; Yang, T.; Zhang, H.; Huang, D.; Wang, K.-H.; Su, Y.; Hu, Y. Chin. J. Org. Chem. 2017, 37, 925 (in Chinese). (李军, 杨天宇, 张怀远, 黄丹凤, 王克虎, 苏瀛鹏, 胡雨来, 有 机化学, 2017, 37, 925.)

(b) Li, J.; Huang, D.; Zhang, H.; Zhang, X.; Wang, J.; Wang, K.-H.; Su, Y.; Hu, Y. Chin. J. Org. Chem. 2017, 37, 2985 (in Chinese). (李军，黄丹凤，张怀远，张兴虎，王娟娟，王克虎，苏瀛鹏，胡 雨来, 有机化学, 2017, 37, 2985.)

(c) Peng, X.; Wang, K.-H.; Huang, D.; Wang, J.; Wang, Y.; Su, Y.; $\mathrm{Hu}, \mathrm{Y}$; Fu, Y. Appl. Organomet. Chem. 2017, 31, 3731.

(d) Du, G.; Huang, D.; Wang, K.-H.; Chen, X.; Xu, Y.; Ma, J.; Su, Y.; Fu, Y.; Hu, Y. Org. Biomol. Chem. 2016, 14, 1492.

[16] (a) Wen, L.; Huang, D.; Wang, K.-H.; Wang, Y.; Liu, L.; Yang, Z.; $\mathrm{Su}, \mathrm{Y}$; Hu, Y. Synthesis 2018, 50, 1979.

(b) Wang, K.-H.; Wang, J.; Wang, Y.; Su, Y.; Huang, D.; Fu, Y.; Du, Z.; Hu, Y. Synthesis 2018, 50, 1907.

(c) Liu, L.; Huang, D.; Wang, Y.; Wen, L.; Yang, Z.; Su, Y.; Wang, K.-H.; Hu, Y. Chin. J. Org. Chem. 2018, 38, 1469 (in Chinese). (刘丽丽, 黄丹凤, 王玉祥, 文岚, 杨政, 苏瀛鹏, 王克虎, 胡雨 来, 有机化学, 2018, 38, 1469.)

(d) Peng, X.; Huang, D.; Wang, K.-H.; Wang, Y.; Wang, J.; Su, Y.; Hu, Y. Org. Biomol. Chem. 2017, 15, 6214.

[17] (a) Formicola, L.; Maréchal, X.; Basse, N.; Bouvier-Durand, M.; Bonnet-Delpon, D.; Milcent, T.; Reboud-Ravaux, M.; Ongeri, S. Bioorg. Med. Chem. Lett. 2009, 19, 83.

(b) Onnis, V.; Cocco, M. T.; Fadda, R.; Congiu, C. Bioorg. Med. Chem. 2009, 17, 6158 .

[18] (a) Diner, C.; Szabó, K. S. J. Am. Chem. Soc. 2017, 139, 2. (b) Carosi, L.; Hall, D. G. Angew. Chem., Int. Ed. 2007, 46, 5913.

(c) Cui, Y.; Li, W.; Sato, T.; Yamashita, Y.; Kobayashi, S. Adv. Synth. Catal. 2013, 355, 1193.

[19] Lade, J. L.; Pardeshi, S. D.; Vadagaonkar, K. S.; Murugan, K.; Chaskar, A. C. RSC $A d v$. 2017, 7, 8011.

[20] Dilman, A. D.; Arkhipov, D. E.; Levin, V. V.; Belyakov, P. A.; Korlyukov, A. A.; Struchkova, M. I.; Tartakovsky, V. A. J. Org. Chem. 2008, 73, 5643.

[21] (a) Hamada, T.; Manabe, K.; Kobayashi, S. Angew. Chem., Int. Ed. 2003, 42, 3927.

(b) Fujita, M.; Nagano, T.; Schneider, U.; Hamada, T.; Ogawa, C.; Kobayashi, S. J. Am. Chem. Soc. 2008, 130, 2914.

[22] Wang, Y.; Wang, K.-H.; Su, Y.; Yang, Z.; Wen, L.; Liu, L.; Wang, J.; Huang, D.; Hu, Y. J. Org. Chem. 2018, 83, 939. 